Mens

Revue d'histoire intellectuelle et culturelle

mens

\title{
Aurélio Ayala et Françoise Le Jeune. Les rébellions \\ canadiennes de 1837 et 1838 vues de Paris, Québec, Les Presses de l’Université Laval, 2011, 218 p.
}

\section{Julien Mauduit}

Volume 13, numéro 1, automne 2012

S’approprier le passé des autres : les usages de l'histoire internationale au Québec avant la Révolution tranquille

URI : https://id.erudit.org/iderudit/1019708ar

DOI : https://doi.org/10.7202/1019708ar

Aller au sommaire du numéro

Éditeur(s)

Centre de recherche en civilisation canadienne-française

ISSN

1492-8647 (imprimé)

1927-9299 (numérique)

Découvrir la revue

Citer ce compte rendu

Mauduit, J. (2012). Compte rendu de [Aurélio Ayala et Françoise Le Jeune. Les

rébellions canadiennes de 1837 et 1838 vues de Paris, Québec, Les Presses de

l’Université Laval, 2011, 218 p.] Mens, 13(1), 150-154.

https://doi.org/10.7202/1019708ar 


\section{Aurélio Ayala et Françoise Le Jeune. Les rébellions cana- diennes de 1837 et 1838 vues de Paris, Québec, Les Presses de l'Université Laval, 2011, 218 p.}

Avec cet ouvrage, l'étude des rébellions franchit pour la première fois le mur de l'Atlantique, ce qui devrait réjouir les historiens québécois et encourager nos collègues nord-américains à reprendre un chantier trop longtemps délaissé. Aurélio Ayala et Françoise Le Jeune offrent l'occasion d'enrichir notre regard sur ces événements grâce à une analyse transatlantique de la crise de 1837-1838, ouverture qui nous amènera à repenser le "nationalisme " attribué aux patriotes.

À l'aide de récits de voyage, de quelques correspondances diplomatiques et de certains journaux, l'ambition des auteurs est de comprendre la réception de la crise canadienne chez les observateurs français, et d'étudier parallèlement l'inaction du gouvernement de Louis-Philippe. L'audace de la proposition consiste à analyser conjointement l'interprétation de la presse parisienne, les réseaux de presse transatlantiques et les relations internationales des années 1830. L'articulation du "libéralisme " et du " nationalisme ", sous l'angle de «l'américanité », sous-tend également l'ambitieuse étude : «Paris verra-t-il dans les rébellions canadiennes une conception civique de la nation chez les patriotes? Au contraire, suivant en cela l'explication du gouvernement britannique, les Français verront-ils un mouvement patriotique, une vision nourrie par leurs affinités identitaires avec les Canadiens? " (p. 13)

Le premier chapitre expose différents récits de voyage. Le conflit canadien est vu tantôt comme un conflit « identitaire " où domine la nostalgie d'une colonie perdue (Alexis de Tocqueville), tantôt comme un combat pour la démocratie, sans distinction entre le Haut- et le Bas-Canada (Isidore Lebrun). Le maintien du régime seigneurial constitue le point d'étonnement majeur pour des Français qui ont connu les bouleversements révolutionnaires : « chose incroyable! rien de tout cela ne figure dans l'interminable liste des quatrevingt-treize griefs, récemment dressée par les Canadiens contre le 
régime qui les gouverne " (Michel Chevalier, à propos des 92 Résolutions, cité p. 33). Le troisième chapitre, sur la diplomatie, révèle l'aversion des autorités françaises pour les rébellions, événements qui renforcent même l'alliance franco-anglaise (p. 138-139). Relevons ici la vision de l'ambassadeur français à Washington qui attribue aux 10 résolutions Russell un caractère "violent " (p. 129), et la reconnaissance par lord Gosford de l'origine de la confrontation armée aux loyalistes (p. 134). Quant aux deuxième et quatrième chapitres, ils portent sur la presse parisienne, étudiée par l'intermédiaire de quatre journaux représentant différents courants politiques. Les feuilles d'opposition, Le Siècle et surtout les républicains du National, défenseurs du "destin civilisateur " de la France, soutiennent quasi quotidiennement les patriotes et appellent à une mobilisation de l'opinion et du gouvernement en leur faveur. À l'inverse, les journaux plus proches du pouvoir ( $L a$ Presse et le Journal des Débats) reprennent globalement les explications des gouvernements alliés. Selon les auteurs, dans la première partie du XIX ${ }^{e}$ siècle, et malgré un intérêt " éphémère " pour le Canada et ses patriotes, le regard français se porte avant tout sur la jeune république voisine, prolongeant « l'ignorance » française envers son ancienne colonie et " américanisant " les patriotes canadiens, c'est-à-dire les intégrant à une histoire continentale dominée par la progression républicaine de l'Union américaine.

Certains choix d'édition, tels que la présentation et le découpage des sections, ajoutés à un enchaînement des arguments parfois maladroit, nuisent toutefois à la lisibilité de la réflexion des auteurs. Il est ainsi écrit qu'en " décembre 1837, les journalistes français découvrent le Canada " (p. 109), mais nous pouvons lire que de nombreux articles sont publiés sur le Canada avant la crise dans $L e$ Siècle et La Presse (p. 85), que le discours de Louis-Joseph Papineau à Saint Laurent est retranscrit en septembre 1837 dans le Journal des Débats (p. 99), et que le National possède un correspondant à New York qui relate la crise dès l'été 1837 (p. 112-113). Évoquons également le concept d' " américanité », critiqué au Québec du fait de son caractère tautologique, qui n’est défini que très partiellement par les 
auteurs (p. 11 et 187). Il apparaît même entrer en contradiction avec l'idée d'une redécouverte de "l'esprit de 1789 » attribuée aux patriotes (p. 166).

Imprécisions et hésitations se retrouvent dans l'analyse du " nationalisme ", problématique au cœur de l'étude, d'autant plus que certaines citations ne soutiennent pas toujours les vues des auteurs. Malgré l'ambivalence historique du terme, ils ne proposent ni définitions ni réflexion méthodologique, ce qui rend confus le lecteur attentif. Les quelques références aux travaux de Marcel Bellavance, qui établit la complémentarité du nationalisme et du libéralisme, composent l'essentiel de la réflexion conceptuelle sur le nationalisme. En introduction, après avoir mentionné que « certains patriotes [...] défendent également les institutions traditionnelles (langue, droit coutumier français, régime seigneurial) ", il est affirmé que "les membres du parti patriote, ayant pour ambition le rôle de classe dirigeante, axent leur programme sur des principes libéraux et démocratiques ainsi que sur la défense et l'émancipation de la "nation canadienne" " (p. 4). La "nation " est ici culturelle et " ethnique ", distincte du politique et référant explicitement à l'héritage de la Nouvelle-France. Or, plus loin, le "nationalisme » du mouvement perd ce sens identitaire pour devenir politique, s'accordant ainsi à son "américanité ", mais contredisant l'affirmation de l'introduction ainsi que la thèse de Bellavance : «il est clair que ce n'est pas dans le clivage ethnique [...] qu'il faut chercher les raisons du soulèvement des Canadas, mais plutôt dans la volonté des patriotes francophones et anglophones d'établir une nation libérale, une nation des Amériques ", inspirée des " méthodes américaines" (p. 116).

La difficulté d'analyser le « national » dans les événements canadiens, puis dans sa réception en France, provient en partie des sens que ce terme prend dans l'histoire. Qu'est-ce qu'une "nation " en 1837 ? Pouvons-nous parler, comme le font les auteurs, de " réveil des nations » en Amérique (p. 115) ? Très éloigné du caractère ethnoculturel contemporain, le National adopte par exemple une définition politique, révolutionnaire : " ce qui distingue la France, ce qui fait 
sa vie et sa nationalité, c'est le besoin de dévouement à la cause de tous ceux qui souffrent [...] La fraternité universelle est sa croyance " (p. 67). L'étude soutient que les journaux d'opposition évoluent vers une vision moins politique et plus romantique de la lutte nationale canadienne, "c'est-à-dire plus française " (p. 161), animée d'une «fibre française » (p. 167). En somme, pour les auteurs, deux conceptions du mouvement s'opposent, celle, politique, de Louis-Joseph Papineau et celle, ethnique, de journalistes parisiens : « les journalistes français sont plus nationalistes que les Canadiens eux-mêmes en 1839 [...] Il y a fort à parier que les journalistes nationalistes français ont dû être "choqués" d'apprendre de la bouche même de Papineau que les Canadiens étaient certes "français d'origine", mais surtout citoyens du Canada " (p. 177). Le terme de " parti français » que les journalistes manient est notamment mentionné pour montrer l'évolution vers ce nationalisme identitaire. Il semble cependant que ce dernier soit toujours synonyme de parti révolutionnaire pour le National, référant plus à la politique qu’à l'ethnicité : « le parti français vit non seulement au Canada, mais encore en Irlande, aux portes mêmes de la vieille Angleterre » (p. 169).

Il ne s'agit pas ici de mettre en doute l'interprétation des auteurs sur la « fibre française » des journalistes parisiens, mais plutôt d'insister sur l'ambivalence propre au terme, car " nation " ne prend une signification réellement ethnoculturelle, en France, qu'à la fin du XIX ${ }^{\mathrm{e}}$ siècle, comme l'a montré Gérard Noiriel dans État, nation et immigration : vers une histoire du pouvoir (Paris, Belin, 2001). L'occasion est ainsi offerte de poser certaines questions sur le nationalisme des patriotes. Le terme " nation ", lorsqu'il est présent dans les rébellions canadiennes, revêt-il son sens politique et révolutionnaire $\mathrm{du} \mathrm{XVIII}^{\mathrm{e}}$ siècle, ce que l'étude d'Ayala et Le Jeune semble suggérer? Et, surtout, comment l'historien doit-il user de ce même terme, défini a posteriori et contenant l'idée plus contemporaine d'" ethnicité » culturelle?

Le livre convaincra malgré tout le lecteur dans son entreprise de désenclaver les événements canadiens pour les intégrer au radicalisme politique transatlantique. L’admiration des républicains français, 
érigeant les Canadiens en symbole, est explicite dans l'étude. Les liens qui sont soulignés entre les radicaux anglais et français confirment, parallèlement, la nécessité de penser l'histoire du politique au XIX ${ }^{e}$ siècle en termes transfrontaliers. Les auteurs insistent en effet sur le rôle des rébellions canadiennes dans l'histoire du républicanisme européen, à l'exemple du National qui saisit l'opportunité de l'élan patriote pour lancer un appel à l'alliance des peuples contre les monarchies (p. 171). Ayala et Le Jeune affirment même que les patriotes canadiens influencent le chartisme anglais; serait-ce là une première hypothèse pour des recherches à venir sur la " communauté d'intérêt " démocratique et transatlantique revendiquée par les radicaux des années 1830 (p. 193-194)?

- Julien Mauduit

Département d'histoire

Université du Québec à Montréal

\section{Alain Lavigne. Duplessis, pièce manquante d'une légende : l'invention du marketing politique, Québec, Éditions du Septentrion, 2012, $194 \mathrm{p}$.}

Maurice Le Noblet Duplessis fut premier ministre du Québec de 1936 à 1939 et de 1944 à 1959. Politicien habile et charismatique, il a marqué son époque. Pourtant, plusieurs aspects du duplessisme demeurent sous-étudiés. En examinant la communication politique de l'Union nationale lors des campagnes électorales de 1936 à 1956, Alain Lavigne s'intéresse à l'un de ces thèmes pour lequel nos connaissances sont relativement limitées. Lavigne est professeur au Département d'information et de communication de l'Université Laval. Comme passion, il collectionne les objets du patrimoine politique québécois, en particulier ceux produits par l'Union nationale durant la période duplessiste. Les objets collectionnés par l'auteur ont été exposés dans différents musées québécois. La plus récente exposition s'est tenue au Musée québécois de culture populaire à Trois-Rivières, sous le titre Duplessis donne à sa province : 